\title{
Study on the Mechanism and Structure of Toothbrush based on Brush Force Control
}

\author{
Yan Luo ${ }^{1, \text { a }}$ Ronglong $\mathrm{Li}^{2, \mathrm{~b}}$ \\ ${ }^{1}$ Guizhou Provincial Light Industry Scientific Research Institute, Guiyang, Guizhou, China, 550003 \\ ${ }^{1}$ Mechanical Engineering School, Guizhou University, Guiyang, Guizhou, China, 550025 \\ aemail, bemail,
}

Keywords: Brush Force, Toothpaste, Brush Head

\begin{abstract}
This paper studies the dental caries, dental calculus deposits, smoke stains, bleeding gums and even lead to gum recession, tooth wedge damage caused by coronary heart disease, diabetes and cerebrovascular diseases due to incorrect brushing posture in daily life. This paper researches the health toothbrush method and health brushing toothbrush brushing mechanism and the structure of the brush force control to get healthy structural principle of the toothbrush.
\end{abstract}

\section{Introduction}

Brush force is too small, not clean brush toothbrush, lead to tooth decay and tartar deposits, smoke stains, etc; brush force is too large, short-term can cause bleeding gums, leading to gum recession long, wedge-shaped tooth damage, but also lead to coronary heart disease, diabetes and cerebrovascular diseases.

Research team at Newcastle University periodontics expert Dr.Giles McCracken and Professor Peter Hersman found that when the brush at 150 grams force, brushing time of 120 seconds, can be most effectively remove plaque to clean teeth when brushing force when more than 150 grams, for further reducing plaque is almost useless.

Dental experts Detroit University School of Dentistry Dr. Luis Litonjua also through the study pointed out that excessive brushing force, even when using a soft-bristled toothbrush, can also cause trauma to the gum tissue, causing gingival recession, gingival recession or make more serious [1].

Experiments by scientists, an adult brush brushing force control in the 0.9 - 1.4 Newton Newton, Newton child controls 0.4-0.45 (Note: 1 Newton equals 0.102 kilograms force), namely: adult brush control force of 100-150 gf, children control at 40-45 between chocolate, can effectively remove plaque without harming the gums, so as to achieve the effect of cleaning teeth gingival care.

\section{The Current State of the Brushing}

Currently on the market whether ordinary toothbrush or electric toothbrush, brush your teeth can not accurately control the force of the brush head. In order to solve the problem of excessive force brush, toothbrush available in the market has done a lot of technological improvements, but these methods only brush the issue of excessive force locally slight improvement, not completely solve the accurate, optimal control brush force technical problems.

At present, the use of the following solutions:

1 , change the bristles soft, rounded bristles head and so on.

The disadvantage is soft bristles change dentifrice reduced ability to remove stubborn stains more easily, more accumulation, smoke debris, plaque, tartar and other generating acceleration.

2, brush both sides of the lining or intermediate care, slowing brush excessive force injury to the gums.

3, or special professional tools. (As shown below) 


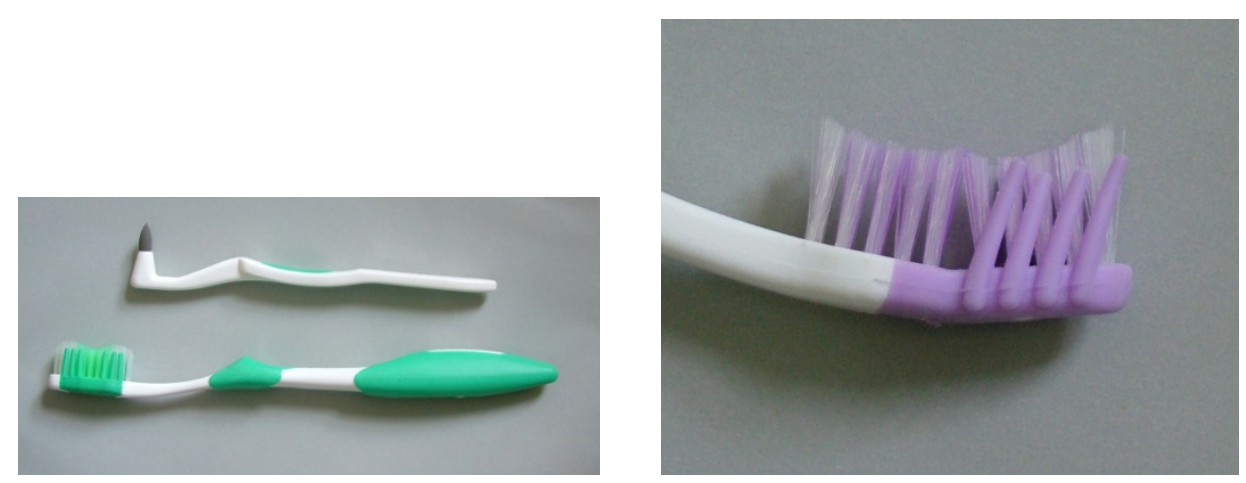

4, The brush handle design elastic structure, decrease impact mitigation and protect the gums.
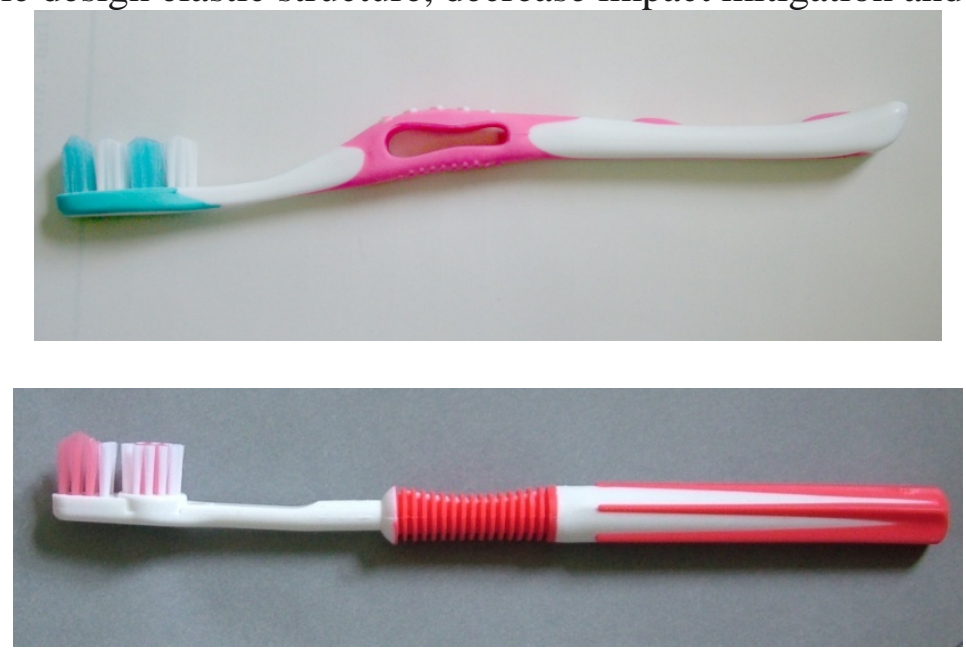

5, By improving the toothpaste, sterilization, whitening to enhance the washing effect.

Because these methods are difficult to accurately control the force of the brush, the dentifrice is also dead, the greater the negative effects brought about, and did not achieve a healthy brushing effect.

\section{Research on Controllable Brush Force Mechanism and Toothbrush Structure}

The end of the toothbrush head and handle separated into two parts, a connection is hinged to the head end with a cam, a spring is connected with one end of the handle DC, and the other end through a flexible cable head end with ED when connected to a soft rope ED fixed at one end of the cam starting point E, one end of the spring DC connection, the cam surface EG spring tension with the head end at the head end of the swing remain tangent, the head end of the swing, spring tension Ft and a hinge point vertical distance $h$ that is the size of the change arm spring tension is controlled by the cam; when the head swing the pendulum the greater the angle $\alpha$, the greater the spring tension Ft, by a cam control the spring tension with the swing angle of arm h the change becomes large, so that the change of the spring and the torque generated by the tension spring tension $\mathrm{Ft}$ and $\mathrm{Nt}$ i.e. the arm spring tension $\mathrm{Ft} \times \mathrm{h}$ by h the product remains constant or within a certain range; when the head end of the oscillating brush head the force $\mathrm{F}$ produces a torque equal to Ns Nt of the torque generated by spring tension, the brush head $\mathrm{l}$ is the lever arm of force to the brush head from the handle end of the hinge point, which is a fixed value by $\mathrm{Ns}=\mathrm{F} \times \mathrm{l}, \mathrm{Nt}=\mathrm{Ft} \times \mathrm{h} \mathrm{Ns}=\mathrm{Nt}$ derived formula

Brush head force $\mathrm{F}=\mathrm{Nt} / \mathrm{l}=\mathrm{Ft} \times \mathrm{h} / \mathrm{l}$

Seen from the formula: the cam and the spring of control, so that $\mathrm{Nt}=\mathrm{Ft} \times$ ht remain constant or within a certain range of variation, $\mathrm{l}$ is a constant, so brush brush force $\mathrm{F}$ in the wobble can be maintained or controlled within a certain range. 


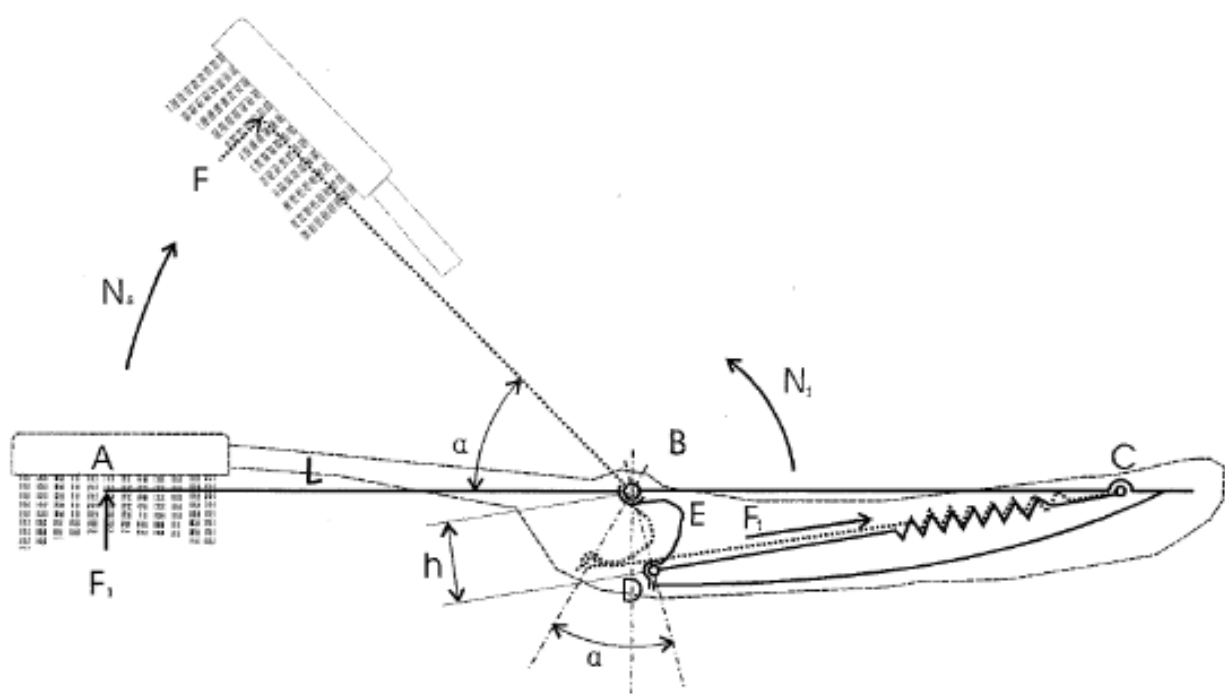

Fig.1 The brush force control mechanism pattern

In addition, according to the brush test date Urso Newcastle University in the UK "Clinical Periodontology" published Xi Siman hosted: tartar increases brushing time and the intensity is reduced, but more than two minutes brushing time, forced more than $150 \mathrm{~g}$ when no corresponding reduction in tartar [2].

Therefore, under normal circumstances, toothbrush structure designed to:

Head swing angle $a$ and brush force $F$ is: $0 \leqslant a \leqslant 90^{\circ}, 100 \mathrm{~g} \leqslant \mathrm{~F} \leqslant 200 \mathrm{~g}$.

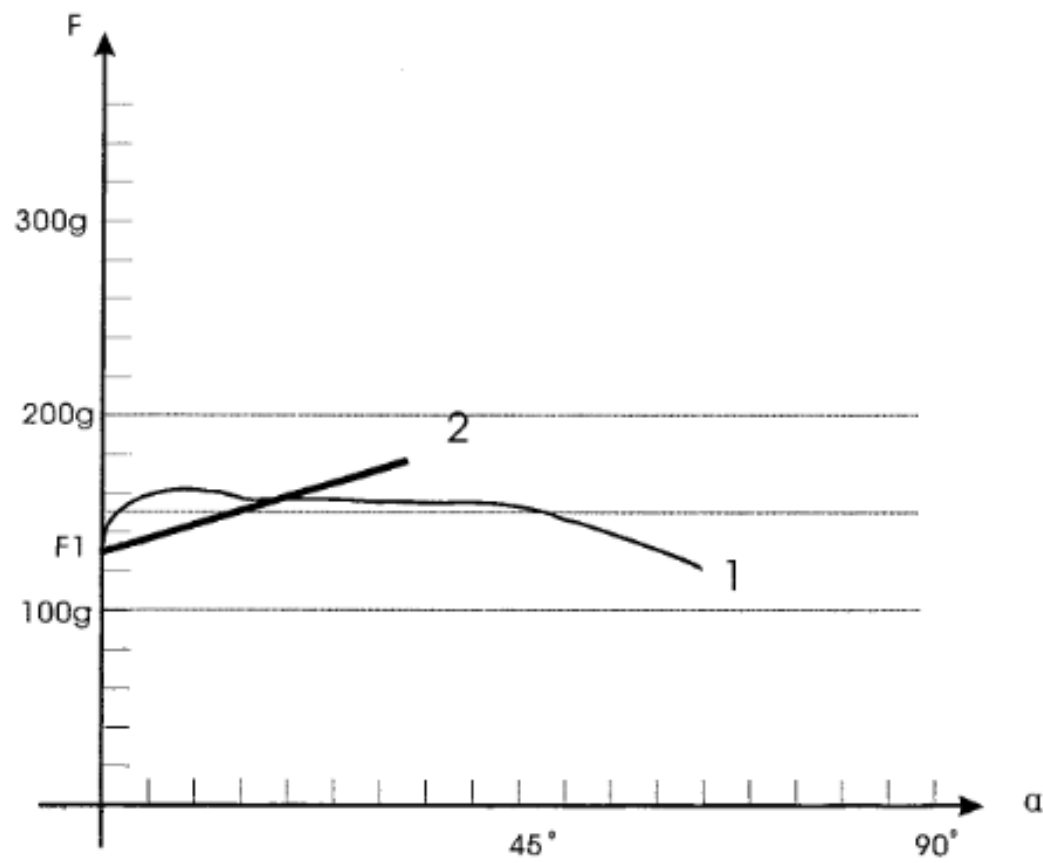

Fig.2 brush head force survey plan

Fig. 2 is to verify that the head of the brush and swing angle $\alpha$ test force $F$ relations made, showing the results from the measurement, in full compliance with the theoretical design.

\section{Conclusion}

Through research, we determine the size of the healthy brushing brush force (such as controlled within $150 \mathrm{~g}$ ). Through the mechanism research of the brush force control, we promote the effective, accurate, reliable control brush force structure and lay a solid theoretical foundation for the development of new health toothbrush. 


\section{References}

[1] Effect of brushing force and time on plaque removal using a powered toothbrush, Journal of Clinical Periodontology, 2003

[2] Tooth brushing and gingival recession, International Dental Journal, April 2003 